\title{
NEURO-FuZZY APPROACH TO MICROCALCIFICATION CONTRAST ENHANCEMENT IN DigitiZED MAMMOgRAM IMAgES
}

\author{
Ayman AbuBaker \\ Department of Electrical and Computer Engineering, Applied Science Private University, \\ Amman, Jordan \\ a_abubakerlasu.edu.jo
}

\begin{abstract}
Computer aided diagnoses can assists radiologists in detecting microcalcification, crucial evidence in mammogram for the early diagnosis of breast cancer. A novel approach is proposed in this paper for early detection of breast cancer by enhancing microcalcification regions in mammogram images using hybrid neuro-fuzzy technique. As a first stage, the mammogram intensities are fuzzified using three linguistic labels. Then, the inference engine of a classical fuzzy system is replaced by a collection of sixteen parallel neural networks and a cascade neural network in order to reduce the computational time for real-time applications. The parallel cascade neural networks are trained using data sets that randomly selected from the original fuzzy decision matrix. Finally, the value of the local mask centre is enhanced after defuzzification the input sets. This work is extensively evaluated using two different types of resources which are Mammographic Image Analysis Society database (MIAS) and University of South Florida (USF) database. As a result, it found to be sensitive in enhancing the microcalcifications regions in mammogram with very little number false positive regions.
\end{abstract}

\section{KEYWORDS}

Mammograms; Microcalcifications; Enhancing MCs, Fuzzy logic, Neuro-fuzzy.

\section{INTRODUCTION}

Breast cancer is one of the most deadly diseases for middle-aged women. One out of eight women is prone to this disease in her lifetime [1]. The success of treatment depends on early detection. Mammogram image (X-ray images of breasts) is the first tool to diagnose the breast cancer. Breast cancer detection on mammograms is currently carried out by radiologists who examine mammograms with a magnifying glass to find out tumours such as microcalcifications (MCs), masses, and stellate lesions [2]. Checking and screening the mammography images remains a difficult process due to poor visualization of MCs and number of cases that needed to be diagnoses. The major reason for poor visualization of small malignant masses is the minor difference in x-ray attenuation between normal glandular tissues and malignant disease [2]. This fact makes the detection of small malignancies problematical, especially in younger women who have denser breast tissue. Although calcifications have high inherent attenuation properties, their small size also results in a low subject contrast [3]. As a result, the visibility of small tumours, and any associated microcalcifications, will always be a problem in mammography as it is currently performed using analogue film. Therefore, the detected clustered of microcalcifications are between $30 \%$ and $50 \%$ of breast cancer cases [3]. For these reasons, computer-aided diagnoses (CAD) are exciting a great deal of attention from the radiologist community $[16,17]$. $\mathrm{CAD}$ is defined as a diagnosis made by a physician taking into account the computer output as a 
The International Journal of Multimedia \& Its Applications (IJMA) Vol.4, No.5, October 2012

second opinion. The goal of applying CAD is to support radiologists' image interpretation and improve the diagnostic accuracy and consistency [16] since automated interpretations of microcalcifications and masses are very difficult due to the regions of interests are usually of low contrast, especially in the case of young women. Therefore, to provide the improved visibility of breast cancer to medical doctors as well as automatic breast-cancer detection systems, mammogram contrast should be enhanced. In doing so, the hybrid neuro-fuzzy enhancement technique is proposed in this paper. The proposed algorithm will accurately enhance the MCs in the mammogram images de-noising is considerable for image enhancement. Especially for mammogram, the size of microcalcification is close to noises. Noise should be reduced while microcalcifications are enhanced. This in case will help the radiologist to accurately detect the $\mathrm{MCs}$ regions in short time.

\section{RELATED WORK}

Many authors had investigated the implementation of mathematical and artificial intelligence methods for enhancing and detecting MCs in mammogram images. One of these techniques, described by Stelios et al. [7], implemented a mathematical morphological and NN approach in order to detect the MCs in the mammogram images. Mathematical morphology provides tools for the extraction of MCs even if they are located on a non-uniform background. For the classification step they employ two types of neural network classifiers, multi-layer perceptron (MLP) and radial basis function neural networks (RBFNN). They found that the performance of the MLP was better than the RBFNN with a sensitivity of $94.7 \%$ TP with $0.27 \%$ false positive per image. Moti and Joskowicz [17] can segment the MCs using the entropy threshold. So, the first step was to remove the background tissues using the multi-scale top hat morphological filtering. Then the entropy threshold based on a third order spatial grey level dependence matrix is applied. They can successfully detect the MCs in the USF database with $93.75 \%$ TP and $6.255 \%$ FP. Lasztovicza et al. [18] introduced a hierarchal neural network to detect the MCs in the mammogram image. In their method, the input image was sampled and the image pyramid is built using multi-scale method. In each resolution level, a number of features was generated and tested using the NN in order to detect the MCs. They can detect the MCs in the miniMIAS database with a TP rate of $90.625 \%$ and a FP rate of $2 \%$ with $9.375 \%$ FN rate. Papadopoulos et al. [20] proposed a hybrid intelligent system to detect MCs in mammogram images. This system was a combination of rule base, ANN and SVM technologies. Their algorithm was implemented to the Nijmegen and MIAS databases. They had $83 \%$ and $81 \%$ TP detection rates for the MIAS and Nijmegen databases, respectively.

Other investigators propose novel neuro-genetic algorithms as tools to detect the MCs in mammogram images. The algorithm of Brijesh and Zhang [12] finds significant features that can be used to extract the MCs from ready marked MC regions in DDSM mammogram images. The feature extraction technique consists of three parts: area extraction from the marked mammograms; feature extraction from the extracted areas; and feature selection for classification for which the neural-genetic algorithm was developed. This algorithm was found to be effective at some threshold values and achieved a maximum TP (selection) of $85 \%$. Peng et al. [14] created a hybrid technique to detect the MCs in mammogram images. The proposed approach was called the knowledge-discovery incorporated genetic algorithm (KD-GA). In this approach, the genetic algorithm (GA) was used to search for bright spots in mammograms and a knowledge-discovery mechanism is employed to improve the performance of the GA. The functions of the knowledgediscovery mechanism include evaluating the possibility of a bright spot being a true $\mathrm{MC}$, and adaptively adjusting the associated fitness values. The adjustment of fitness is used as an indirect guide for the GA to extract the true MCs and to eliminate the false MCs. Therefore, the performance of the algorithm in detection the MCs was slightly improved when using KD-GA approach. So, the KD-GA approach was effectively detect the MCs by $98.9 \%$ TP but with high detected FP clusters which is $40 \%$. Also Jiange et al. [21] proposed a GA algorithm in detecting 
The International Journal of Multimedia \& Its Applications (IJMA) Vol.4, No.5, October 2012

the MCs in mammogram images. Their algorithm was based on transforming input images into a feature domain, where each pixel is represented by its mean and standard deviation. The chromosomes are constructed to populate the initial generation and further features are extracted to enable the proposed GA to search for optimized classification and detection of microcalcification clusters. As a result, their algorithm had sensitivity of $91 \%$ with specificity 95\% when processing the whole mammogram image. But, the processing time needed to process the whole mammogram images was 15 minutes.

\section{DATABASE RESOURCES}

In this work, the MC enhancing algorithm is trained and tested on 190 mammographic images from the University of South Florida (USF) and MIAS databases (140 from USF and the remainder from MIAS). The USF database is a publicly available digital database for mammography screening. Its images are collected from different medical schools and hospitals across the USA. These images all have the same specification (3000 pixel $\times 4500$ pixel and 16-bit pixel depth). This database is divided into four volumes representing the different types of diagnosis: normal, cancer, benign, and benign without call back. Normal images are from patients with normal examination results that have had normal examinations in the previous four years. A normal screening examination is one in which no further "work-up" is required. Cancer images are from patients with screening examinations in which at least one pathology proven cancer is found. Benign cases are from patients with screening examinations in which something suspicious was found, which turned out to be non-malignant (by pathology, ultrasound or some other means). The term benign without call-back is used to identify benign cases in which no additional X-rays or biopsies were done. In this paper $70 \mathrm{MCs}$ mammogram images are collected from seven cancer volumes and 70 normal mammogram images are collected from four normal volumes. The cancer volumes are: cancer_01, cancer_05, cancer_06, cancer_07, cancer_13, cancer_14, and cancer_15. The normal volumes are: normal_02, normal_05, normal_07 and normal_09.

The MIAS mammograms have been carefully selected from the United Kingdom National Breast Screening Program. The 322 images represent 161 patients in the MIAS database. These images have been expertly diagnosed and the positions of the MCs in each image are recorded. In this paper, $25 \mathrm{MC}$ and 25 normal additional mammogram images were selected from the MIAS database. The mammograms in this database were obtained using the medio-lateral oblique (MLO) view and were digitized at a spatial resolution of $0.05 \mathrm{~mm}$ pixel size with 8-bit density resolution. Four image sizes, corresponding to different breast sizes, are included in the 322 images from 161 patients: small $(4320$ pixel $\times 1600$ pixel $)$, medium $(4320$ pixel $\times 2048$ pixel $)$, large $(4320$ pixel $\times 2600$ pixel $)$ and extra-large $(5200$ pixel $\times 4000$ pixel $)$. Digitization was performed on a Joyce-Loeble scanning microdensitometer (SCANDIG-3) which has a linear response in the range 0.0 to 3.2 optical densities.

\section{NEURO-FUZZY ENHANCEMENT APPROACH}

The neuro-fuzzy enhancement technique (NFET) is divided to four major stages as shown in Figure 1. The following section introduced detailed description for each stage while the next section introduces the problems exists in paper [30] and how it is overcome in this approach. 


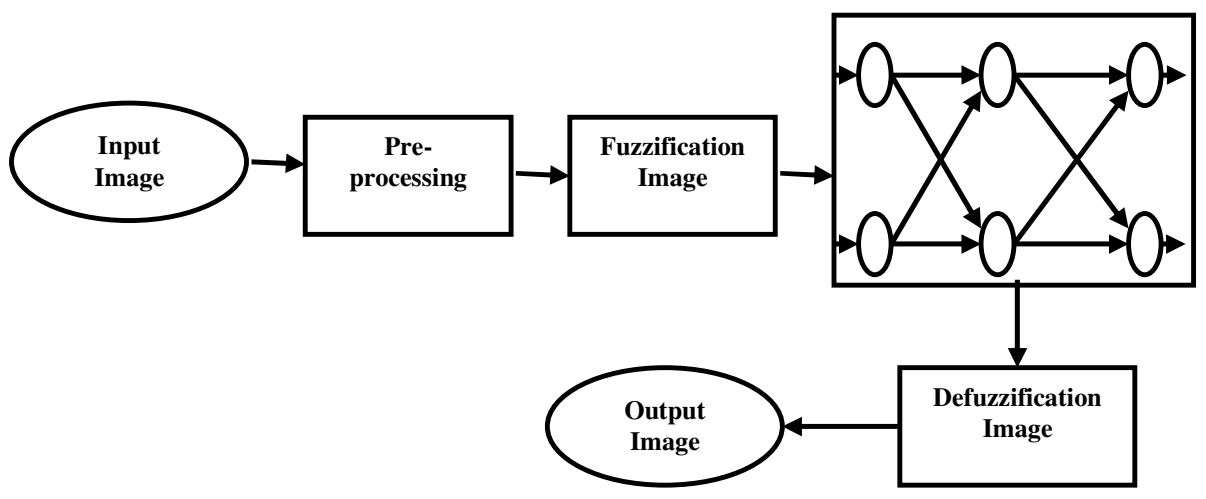

Figure 1. NFET flowchart

\subsection{Enhancement Fuzzy Logic Technique}

The enhancement fuzzy logic technique (EFLT) was analysed and tested for different mammogram cases based on the same parameters and rules used by Abubaker [30]. The enhancement method results have been considered with relation to different mammogram cases. Figures from 2 to 5 present different cases that EFLT is produce such as missing some MC cases and enhancing the false positive regions. The large processing time, missing some MCs regions, and enhancing large number of false positive regions were the main problems in the EFLT approach. The reasons behind the failure in enhancing the MCs regions accurately in the 190 mammogram images were related to the limited mask size that is used in his work which is $3 \times 3$ mask size and their eight connected neighbours. All these problems are tackled and maintain in the novel neuro-fuzzy enhancement technique that will be introduce in the following sections.

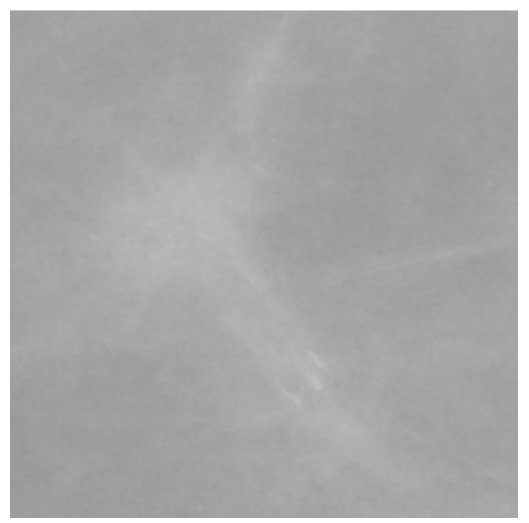

(A)

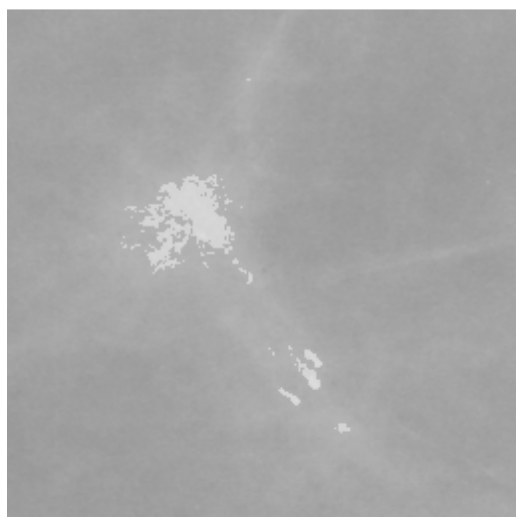

(B)

Figure 2. (A) Original MC Image (B) Poor MC Enhancement 
The International Journal of Multimedia \& Its Applications (IJMA) Vol.4, No.5, October 2012

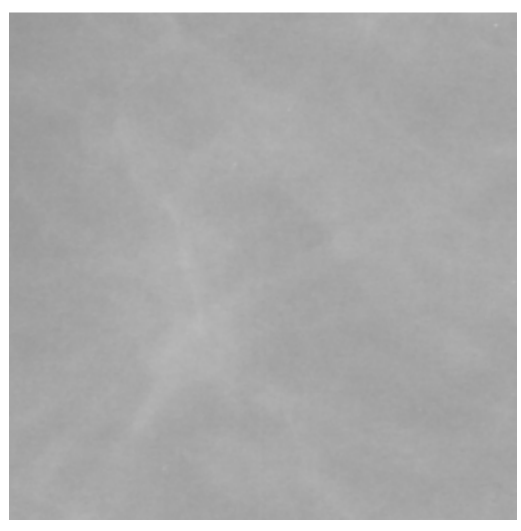

(A)

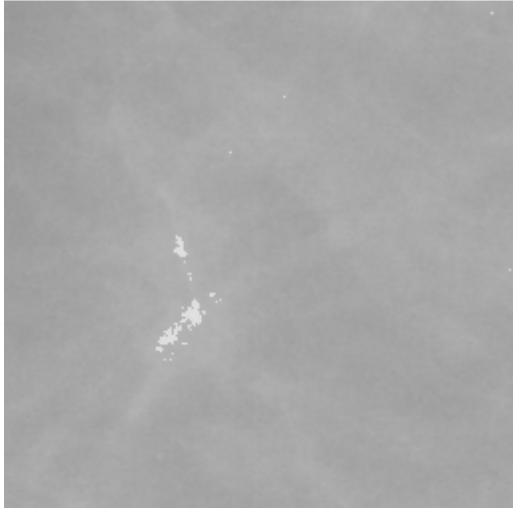

(B)

Figure 3. (A) Original MC Image (B) Poor line MC Enhancement

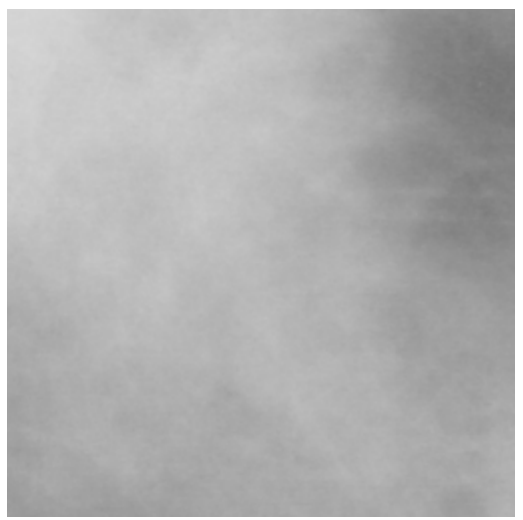

(A)

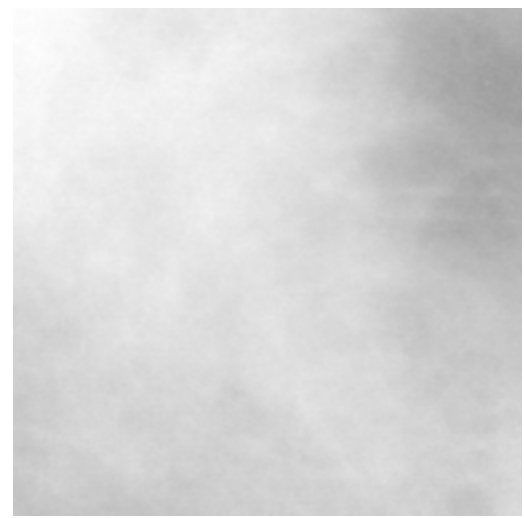

(B)

Figure 4. (A) Original MC Image (B) Poor MC Enhancement

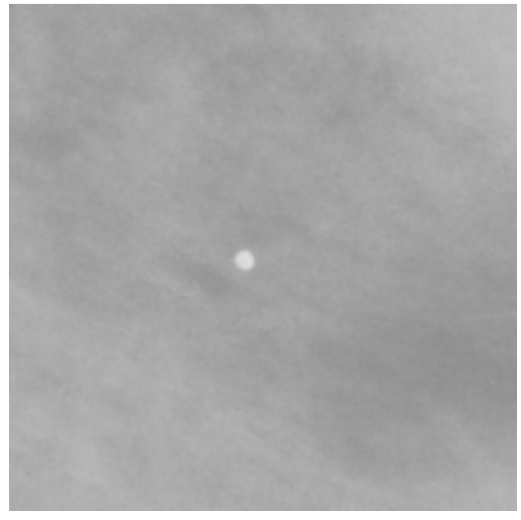

(A)

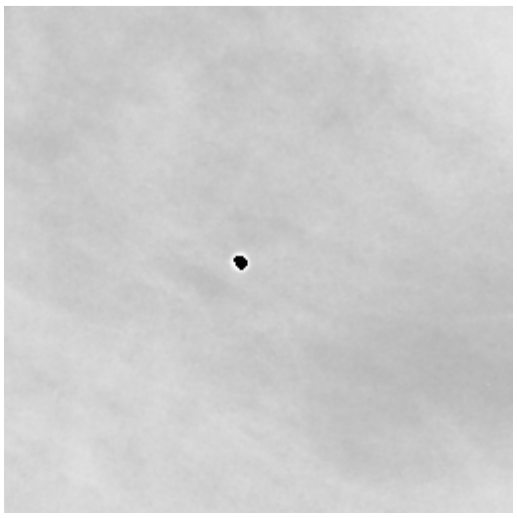

(B)

Figure 5. (A) Original Normal Image (B) False positive Enhancement 
The International Journal of Multimedia \& Its Applications (IJMA) Vol.4, No.5, October 2012

\subsection{Pre-processing Stage}

The pre-processing stage is used to remove the artefacts that exist in the mammogram images as in [30]. These artefacts are cased from capturing images on X-ray film and the process used to digitize the images. These artefacts are removed based on the fact that MCs areas in mammogram images are hazy regions. Conducted extensive analysis of 190 mammogram images from the USF and MIAS databases is carried out and concluded that all MCs have grey scale values in the range from 40 to 240. In accordance with these observations, each mammogram is analysed to determine more precisely: an upper limit threshold (UT) and a lower limit threshold (LT). These thresholds are used to exclude the artefacts within the breast region and are determined based on the statistical characteristics of every mammogram. An upper threshold value (UT), is set to 240 to eliminate the bright artefact regions. The lower threshold value (LT) is calculated from the mean $\mu 0$ and standard deviation $\sigma 0$ values of all non-zero pixel values $x_{i, j}^{0}$, as shown in equation (1). Since there are different types of breasts such as normal, fatty and dense breasts, it was found that using this equation provides better performance compared to using the mean value only, which will eliminate MC clusters from dense breasts in particular.

$$
L T=\mu_{0}-\sigma_{0}
$$

Applying a lower threshold LT helps to eliminate the background regions and low intensity pixel artefacts, and to eliminate the low-level boundary regions of the breast, hence reducing the size of the regions of interest. These two limits are applied as the algorithm scans the breast region to enhance the potential MCs in the mammogram images.

\subsection{Fuzzification Stage}

As it has been already noted, that the EFLT in [30] is not capable to enhance accurately all the mammogram images. The main reason behind that failure is the mask size. MCs appear in digitized mammograms as small regions, with intensity values higher than the surrounding region. The size of MCs is usually less than $1 \mathrm{~mm}[13,26]$. The size of the mask in pixels should be determined based on the resolutions of the USF and MIAS databases which are $45 \mu \mathrm{m} \times 45$ $\mu \mathrm{m}$ and $50 \mu \mathrm{m} \times 50 \mu \mathrm{m}$ respectively. Therefore, the mask size is set to be $5 \times 5$ is appropriate to initially include the peak of the MC regions.

In this stage, all the intensities in the image is translated into fuzzy linguistic labels (Low, Medium, High) with membership values $\left(\mu_{L}(x), \mu_{M}(x), ..\right)$ within a universe of discourse of [LT to UT] grey levels for each input intensity as shown in Figure 6.The membership function of a fuzzy set maps all input intensities of the set into real numbers in $[0,1]$. The larger values of the membership represent the higher degrees of the belongings. In this paper two commonly used membership functions are used for a grey level image which are trapezoid and triangular membership functions as presented in equations 2 and 3 respectively. 
The International Journal of Multimedia \& Its Applications (IJMA) Vol.4, No.5, October 2012

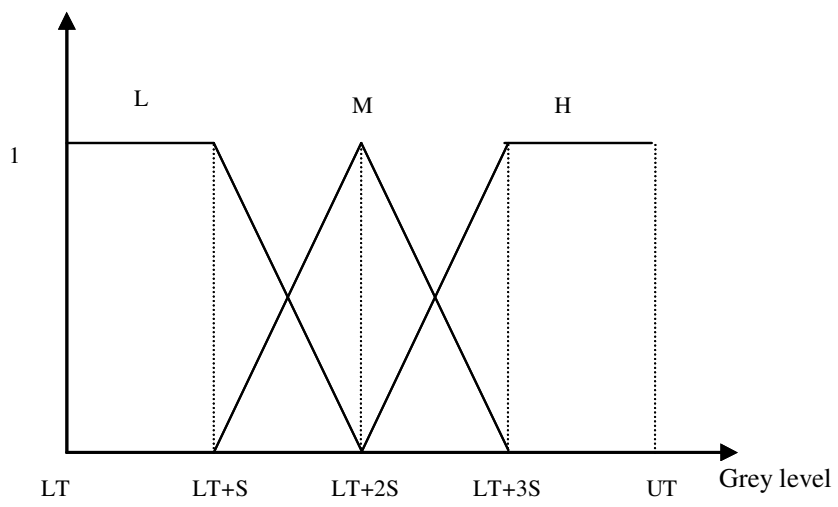

Figure 6. Fuzzification input image intensities

$M(X, a, b, c, d)= \begin{cases}0 & \mathrm{X} \leq \mathrm{a} \\ \frac{(X-a)}{b-a} & \mathrm{a} \leq X \leq \mathrm{b} \\ 1 & b \leq X \leq c \\ \frac{(\mathrm{d}-\mathrm{X})}{(c-b)} & \mathrm{c} \leq X \leq d \\ 0 & \mathrm{X} \geq d\end{cases}$

$M(X, a, b, c)= \begin{cases}0 & \mathrm{X} \leq \mathrm{a} \\ \frac{(X-a)}{b-a} & \mathrm{a} \leq X \leq \mathrm{b} \\ \frac{(c-\mathrm{X})}{(c-b)} & \mathrm{b} \leq X \leq \mathrm{c} \\ 0 & \mathrm{X} \geq \mathrm{c}\end{cases}$

The parameters a, b, c which determine the shape of the trapezoid and triangular membership functions are dynamically sets based on the LT and UT for each mammogram image. These parameters are changes for different mammogram images that have different topologies. The step (S) in the fuzzification engine is calculated based on the equation 4.

$S=\frac{U T-L T}{4}$

\subsection{Neural Network Stage}

In the inference fuzzy stage, a mask of size $5 \times 5$ is used to select number of inputs for the inference engine as shown in Figure 7. The location of the central pixel of the mask is chosen as a reference point and the sixteen connected neighbours will be inputs for the inference engine. The mask convolution for the whole mammogram pixels in the range between the LT and UT are processed in the inference engine as sixteen inputs for each convolution step. The number of the rules to construct the rule-base depends on the number of inputs $(n)$ and the number of fuzzy sets allocated to each input $(m)$. Consequently, the number of rules required is $m^{n}$. Therefore number of rules in the inference engine is $3^{16}=43,046,721$ activation rules. 


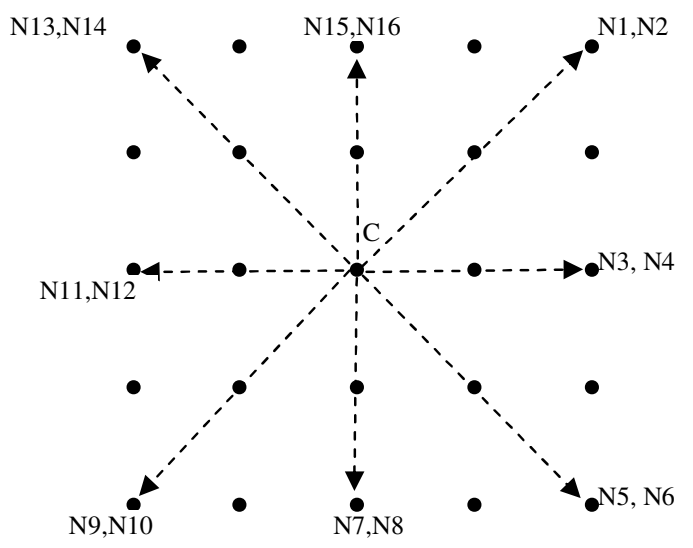

Figure 7. 16-connected neighbours for the mask $5 \times 5$.

The main problem in EFLT presented in reference [30] is the inference block, which consists of a large number of rules (6561 activation rules) that need a long processing time. So, increasing number of rules from 6561 to $43,046,721$ activation rules will sharply increase the processing time for the mammogram images. To solve this problem, the inference engine was replaced with a neural network. The system is investigated by considering the results of the integration between both systems (Fuzzy logic and neural networks).

\subsubsection{Creating the Input and the Output Data}

The neural network training in the feedforward method, the inputs, and the outputs data must be known. The inputs data are the fuzzy linguistic labels from the fuzzification block and the fuzzification block takes the crisp inputs from image intensities. As shown in Figure 6, the intensity range is [LT, UL] grey levels, so the representative sample for each input that is used to train and test the neural network is design based on the equation 5 .

$$
\text { Number_Of_Samples_For_Each_Input }=\frac{U T-L T}{2}
$$

On average, the LT and UT for most mammogram image were in the range [60 to 240] grey levels. So the resulted matrix for each input will be 90 rows with three columns corresponding to the fuzzy sets of the input. As a result of the combinations of all these sixteen matrixes, the input matrix of the neural network will be 18530201888518410000000000000000 rows with 48 columns where the this number comes from the combination of the all matrix rows $90 \times 90 \times$ $90 \ldots \ldots \ldots \times 90=18530201888518410000000000000000$ and the number 48 comes from the sum of all the inputs sets $3+3+3 \ldots+3=48$. To find the output of this huge numbers $18530201888518410000000000000000 \times 48$ matrix that cannot be processed using feedforward neural network. Therefore, cascade parallel neural network is used to train the inputs.

\subsubsection{Training the Neural Network}

As has been shown in the previous section, it is clear that the input data is very large and the learning process for this type of data is so difficult using feedforward neural network with structure (48 input nodes and 5 output nodes). The maximum output error for this neural network topology will be always high. To overcome this, the cascade parallel neural network is used. The multi neural network is used in this work where the input layer consists of 3 nodes and the output layer consists of five nodes with sixteen copies of these neural networks as number of inputs is 
The International Journal of Multimedia \& Its Applications (IJMA) Vol.4, No.5, October 2012

16. Then the output of the each neural network is connected to the cascade neural network where it has 48 nodes in the input layer and 5 output nodes for the output layer as shown in Figure 8.

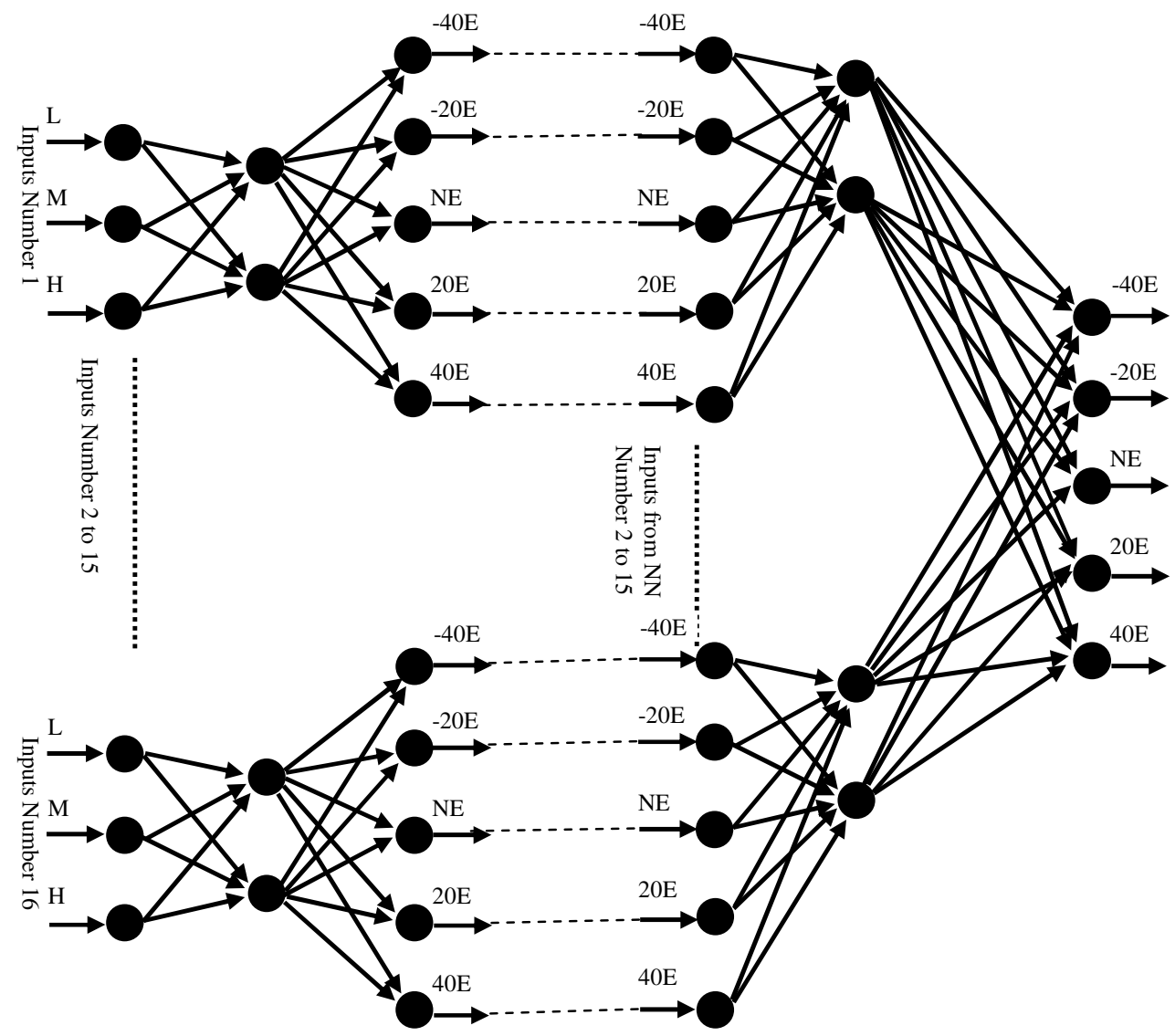

Figure 8. Cascade Parallel Neural Network

\subsubsection{Learning Process}

Initially, 16 input matrices of size 90 rows and 3 columns are generated based on equation (5). The intensity value for each input matrix is shifted by 2 grey levels comparing with the next input matrix in order to have different combination for the input matrices. The learning process for the 16 parallel neural networks is carried out as individual nets. So, each one of the input matrices is processed in its desired neural network. On the other hand, the cascade neural network is processed using an input matrix of that is resulted from the parallel neural network that have a size 90 rows with 48 columns.

The values of the input matrices are arranged in training vectors in a manner similar to the Jackknife technique [29], where 63 grey levels were used for the NN training phases and the remaining 27 were used for the $\mathrm{NN}$ testing phases.

The topology of the each of the NN consists of three input nodes (Low, Medium, High), hidden layer and five output node $(-40 \mathrm{E},-20 \mathrm{E}, \mathrm{NE}, 20 \mathrm{E}, 40 \mathrm{E})$. To find the optimum topology (i.e., the optimum number of hidden nodes in the hidden layer), several feedforward $\mathrm{NN}$ structures were trained using the training data to find the minimum training error, as shown in Figure 9. As a result, it is found that the feedforward NN with one hidden layer and nine nodes for the parallel 
The International Journal of Multimedia \& Its Applications (IJMA) Vol.4, No.5, October 2012

$\mathrm{NN}$ and feedforward $\mathrm{NN}$ with one hidden layer and seventeen nodes for the cascade NN produce the minimum errors.

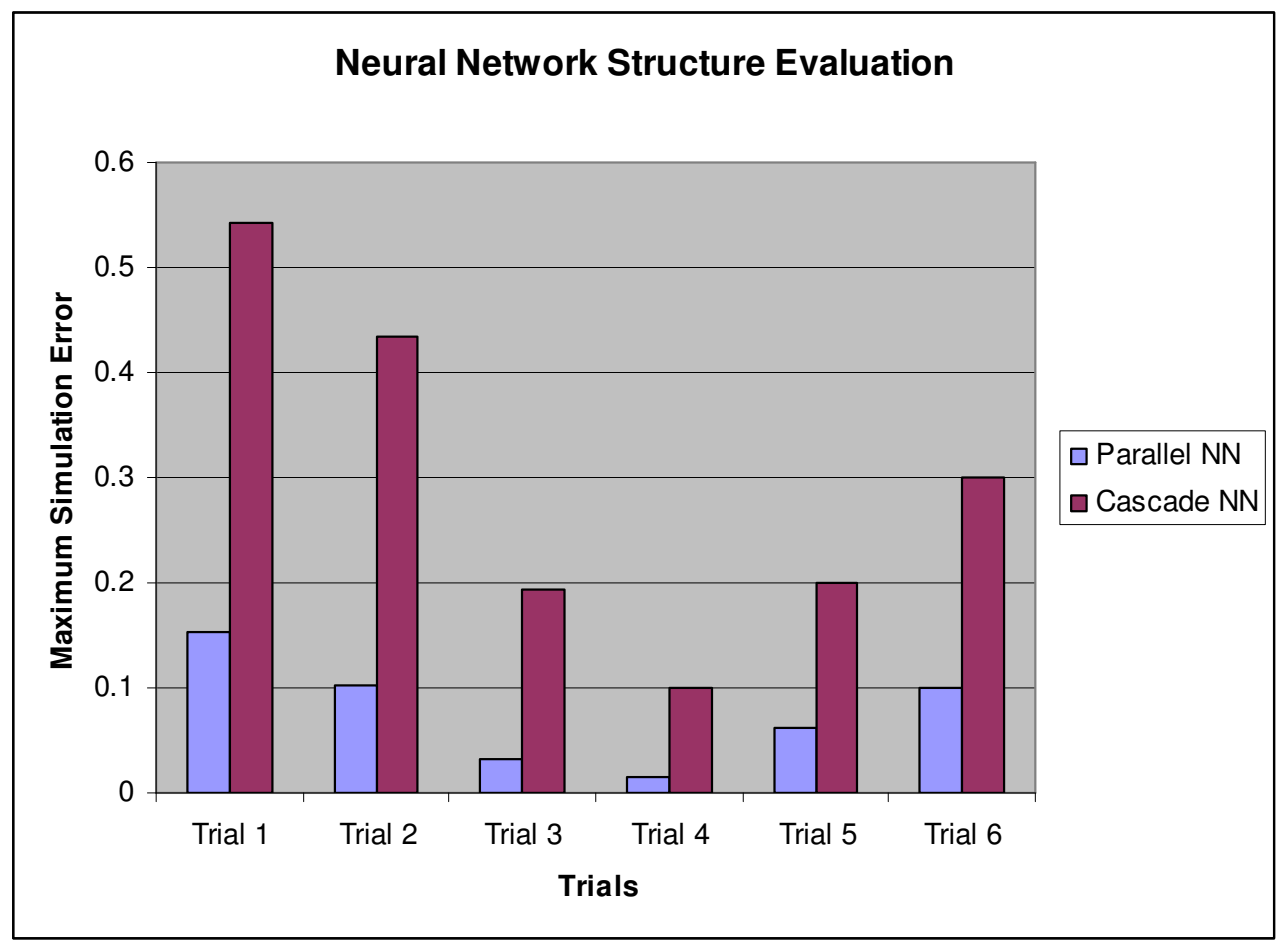

Figure 9. Neural network Structure Evaluation, where, Trial 1 (Parallel NN: one hidden layer with 3 nodes, Cascade NN: one hidden layer with 3 nodes), Trial 2 (Parallel NN: one hidden layer with 5 nodes, Cascade NN: one hidden layer with 5 nodes), Trial 3 (Parallel NN: one hidden layer with 7 nodes, Cascade NN: one hidden layer with 11 nodes), Trial 4 (Parallel NN: one hidden layer with 9 nodes, Cascade NN: one hidden layer with 17 nodes), Trial 5 (Parallel NN: two hidden layers with 7, 5 nodes, Cascade NN: two hidden layers with 17, 5 nodes), Trial 6 (Parallel NN: two hidden layer with 11, 5 nodes, Cascade NN: two hidden layer with 20,10 nodes).

\subsection{Defuzzification Stage}

The output of the cascade parallel neural network is five nodes which are the defuzzification sets. In this paper, the defuzzification is represented as five linguistic labels $(-40 \mathrm{E},-20 \mathrm{E}, \mathrm{NE}, 20 \mathrm{E}$, $40 \mathrm{E})$. The universe of discourse for the fuzzy set is dynamic based on the intensity value of local mask centre. The universe of discourse is set to be $(-50 \%$ to $+50 \%$ of the intensity value of mask centre) as shown in Figure 10. 


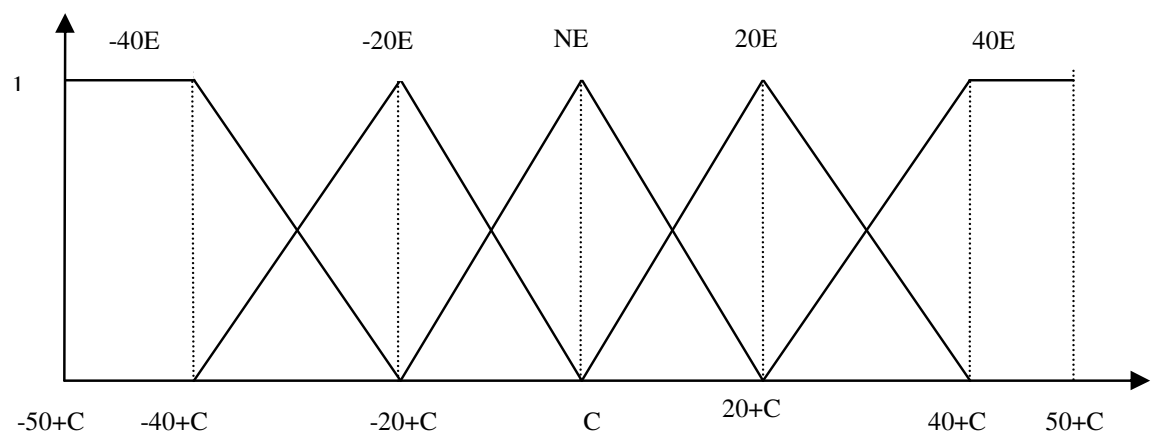

Figure 10: Defuzzification output sets

\section{Evaluation The Proposed Algorithm}

Different mammogram images from the USF and MIAS databases are used in this evaluation process. The processed images are subjectively compared with pre-diagnosis cases for the mammogram images from the USF and MIAS databases in order to classify the enhanced regions into TP and FP clusters. Figures from 11 to 15, present the accurate enhancement for the main five types of the MCs in the mammogram images which are linear, lobular, powdery, angular and vascular MC shapes. Processing time for the mammogram image is another challenge that is considered in this evaluation.

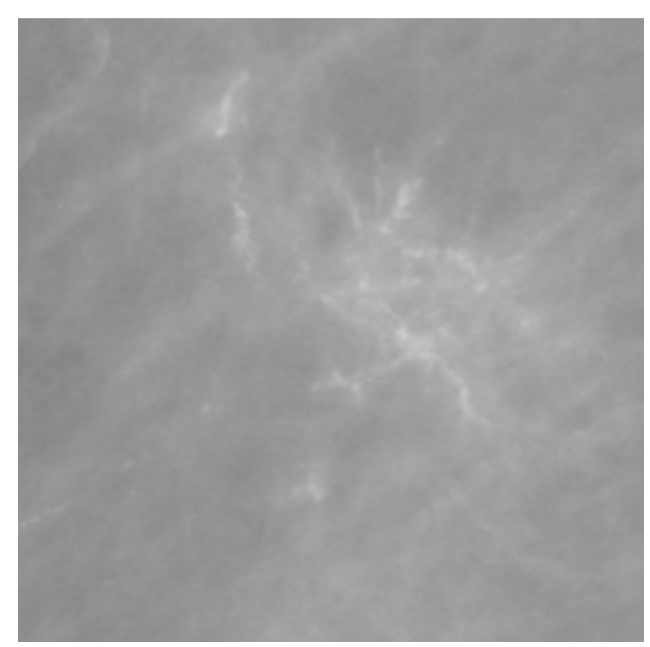

(A)

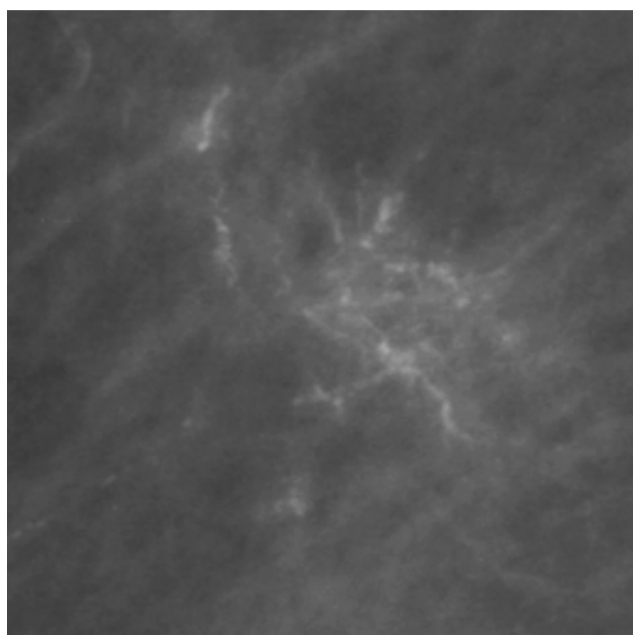

(B)

Figure 11. (A) Linear MC image (B) Processed Linear MC image. 
The International Journal of Multimedia \& Its Applications (IJMA) Vol.4, No.5, October 2012

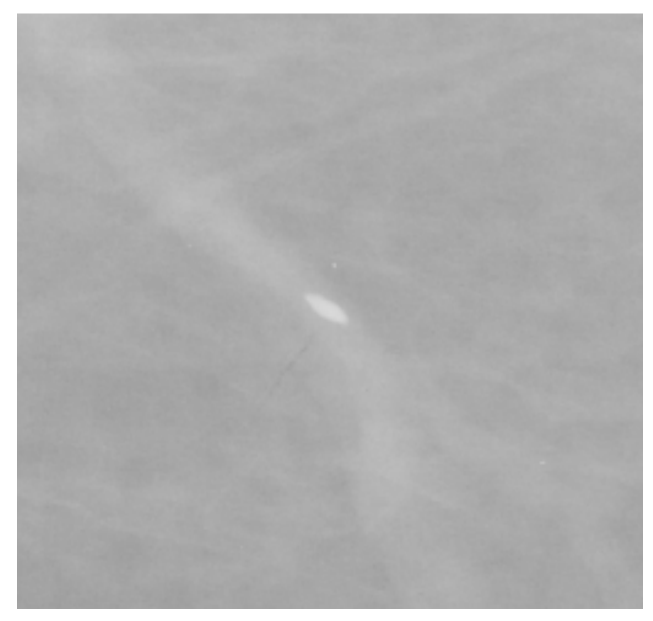

(A)

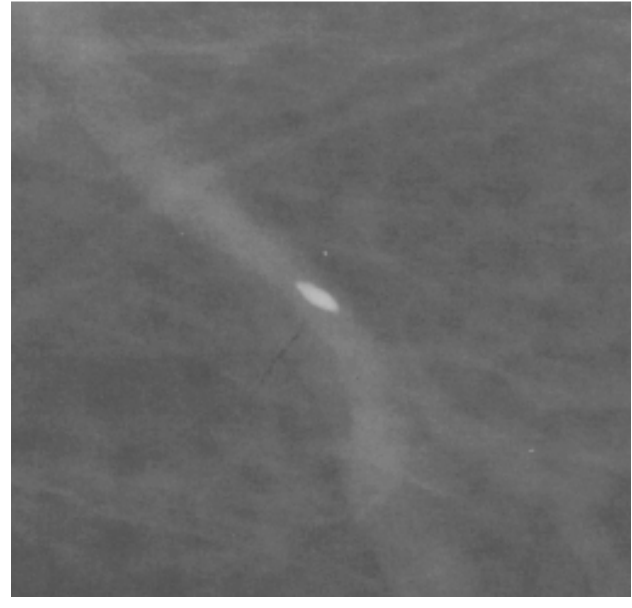

(B)

Figure 12. (A) Vascular MC image (B) Processed Vascular MC Image.

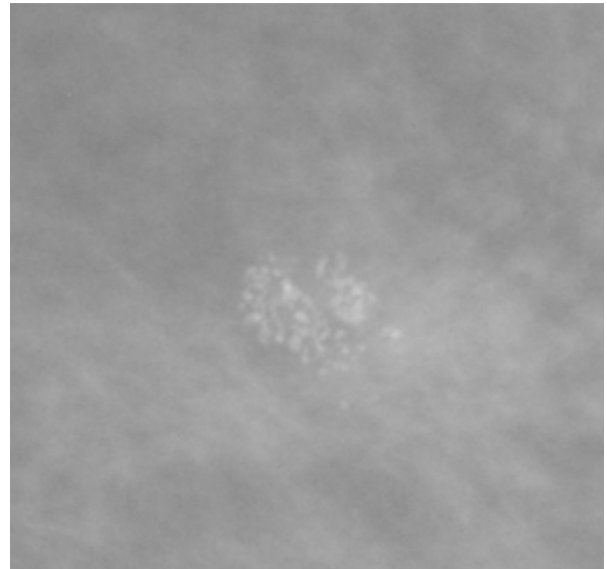

(A)

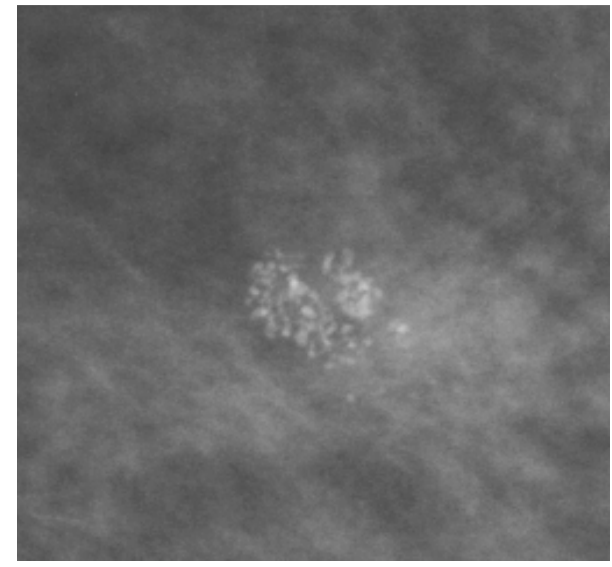

(B)

Figure 13. (A) Powdery MC image (B) Processed Powdery MC Image.

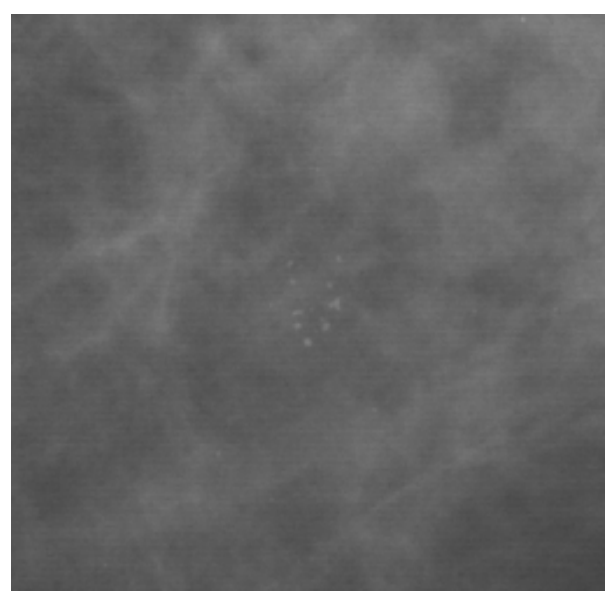

(A)

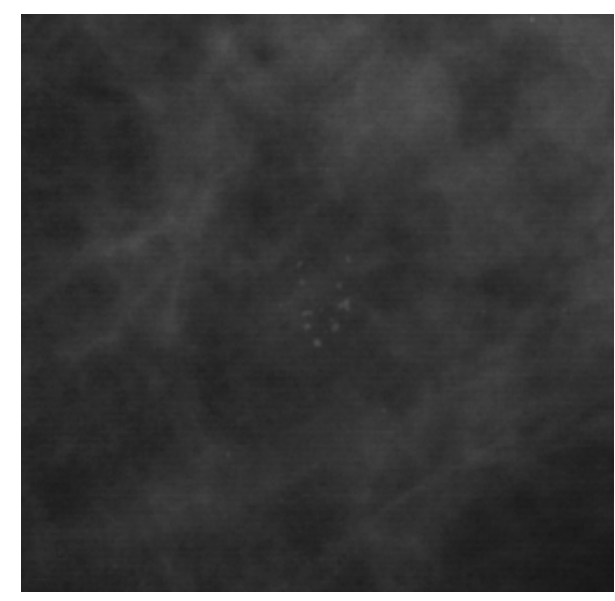

(B)

Figure 14. (A) Lobular MC image (B) Processed Lobular MC Image. 

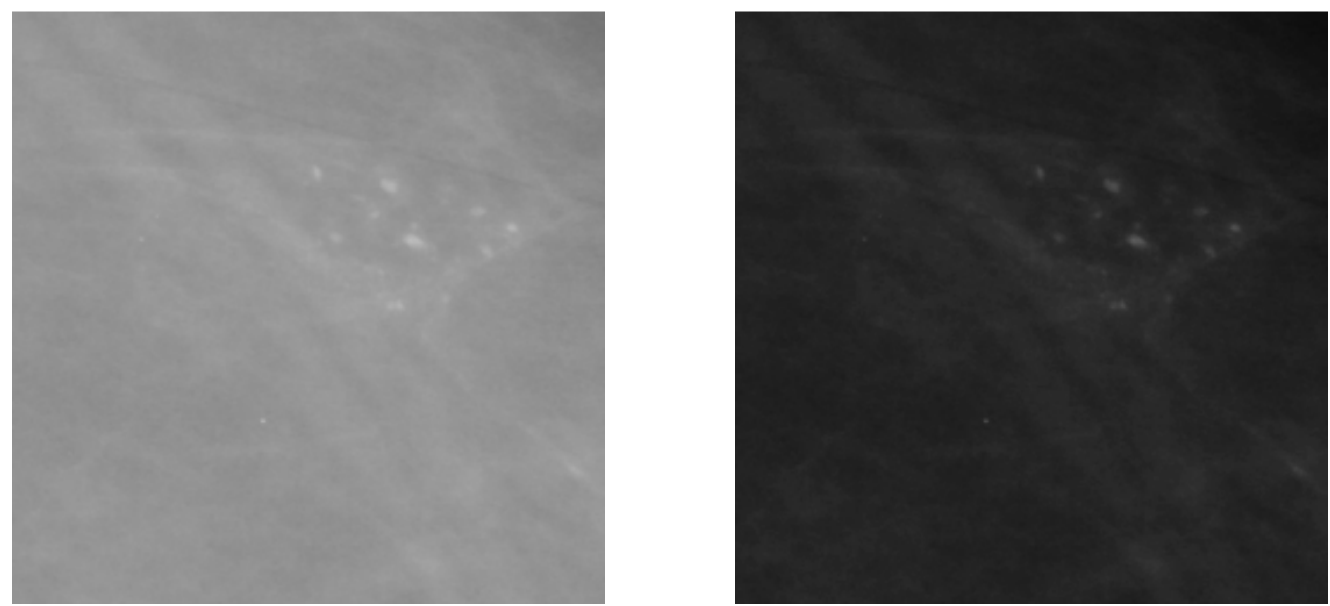

Figure 15. (A) Angular MC image (B) Processed Angular MC Image.

The most important advantage gained by utilizing the hybrid neuro-fuzzy approach in enhancing the MCs in mammogram images that reduce the processing time needed to execute 43,046,721 activation rules to be processed in $201 \mu \mathrm{S}$ which increases the performance of the proposed CAD system in detecting and enhancing the MCs in the mammogram images.

\section{CONCLUSiOnS}

This paper present a novel approach to enhance the MCs in mammogram images accurately with minimum number of false positive regions using hybrid neuro-fuzzy technique. The first stage was fuzzification all the pixels in the mammogram images based on number of input fuzzy sets. Due to the huge number of activation fuzzy rules in the inference system which will increase the processing time, a cascade parallel neural network is proposed. Design and learning the neural network is accurately set by using different neural networks topologies with representative input samples. Finally, the output of neural network is converted to crisp intensity using defuzzification stage.

The proposed algorithm can accurately enhance the MCs in the mammogram images with minimum number of false positive regions which will increase the performance CAD system in diagnoses the MCs in the mammogram images.

\section{REFERENCES}

[1] P. Sakellaropoulos, L. Costaridou, G. Panayiotakis. A wavelet-based spatially adaptive method for mammographic contrast enhancement. Phys. Med. Biol. Vol. 48, pp. 787-803, (2003).

[2] S.G. Chang, B. Yu, M. Vetterli. Adaptive Wavelet Thresholding for Image Denoising and Compression. IEEE Trans. Image Processing, Vol. 9, No. 9, pp.1532-1546, (2000).

[3] J.H. Yoon, Y.M. Ro. Enhancement of the Contrast in Mammographic Images Using the Homomorphic Filter Method. IEICE Transactions on Information and Systems, Vol.85-D, No.1, pp. 291-297, (2002).

[4] Valliappan Raman, Putra Sumari. Digital mammogram segmentation: an initial stage. ACST '08 Proceedings of the Fourth IASTED International Conference on Advances in Computer Science and Technology, 259-263, (2008)

[5] S. Setarhedan, and S. Singh, Eds. London, U.K. Springer-Verlag, pp. 440-540, (2001).

[6] Sameer Singh, Reem Al-Mansoori. Identification of regions of interest in digital mammograms, Journal of Intelligent Systems, Vol. 3, pp. 230-238, (2000). 
The International Journal of Multimedia \& Its Applications (IJMA) Vol.4, No.5, October 2012

[7] Stelios Halkiotisa, Taxiarchis Botsisa, Maria Rangoussi. Automatic detection of clustered microcalcifications in digital mammograms using mathematical morphology and neural. Networks Signal Process, Vol. 1, pp.476-483, (2007).

[8] R. G. Bird, T. W. Wallace, and B. C. Yankaskas. Analysis of cancers missed at screening mammography. Radiology, vol. 184, pp. 613-617, (1992).

[9] H. Burhenne, L. Burhenne, F. Goldberg, T. Hislop, A. J. Worth, P. M. Rebbeck, and L. Kan. Interval breast cancers in the screening mammography program of British Columbia: Analysis and classification,” Am. J. Roentgenol. vol. 162, pp. 1067-1071, (1994).

[10] K. Doi, H. MacMahon, S. Katsuragawa, R. M. Nishikawa, and Y. Jiang. Computer-aided diagnosis in radiology: Potential and pitfall. Eur. J. Radiol., vol. 31, pp. 97-109, (1999).

[11] A. F. Laine, J. Fan, andW. Yang. Wavelets for contrast enhancement of digital mammography. IEEE Eng. Med. Biol. Mag., vol. 14, no. 5, pp.536-550, (1995).

[12] Brijesh Verma, Ping Zhang. A novel neural-genetic algorithm to find the most significant combination of features in digital mammograms. Applied Soft Computing, Vol. 7, pp. 612-625, (2007).

[13] Marius George Linguraru , Kostas Marias, Ruth English , Michael Brady. A biologically inspired algorithm for microcalcification cluster detection. Medical Image Analysis Vol. 10, pp. 850-862, (2006).

[14] Yonghong Peng, Bin Yao, Jianmin Jiang. Knowledge-discovery incorporated evolutionary search for microcalcification detection in breast cancer diagnosis. Artificial Intelligence in Medicine, Vol. 37, pp. 43-53, (2006).

[15] Ping Zhang, Brijesh Verma, Kuldeep Kumar. Neural vs. statistical classifier in conjunction with genetic algorithm based feature selection. Pattern Recognition Letters Vol. 26, pp. 909-919, (2005).

[16] S. Sentelle, M. Sentelle and M.A. Sutton. Multiresolution-Based Segmentation of Calcifications for the Early Detection of Breast Cancer. Real-Time Imaging, Vol. 8, pp. 237-252, (2002).

[17] Moti Melloul and Leo Joskowicz. Segmentation of microcalcification in X-ray mammograms Using entropy thresholding. CARS- Springer, Vol 6, 1-5, (2002).

[18] László Lasztovicza, Béla Pataki, Nóra Székely, Norbert Tóth. Neural Network Based Microcalcification Detection in a Mammographic CAD System. International Scientific Journal of Computing Vol.3, pp. 1727-6209, (2004).

[19] Thor Ole Gulsrud and John Håkon Husøy. Optimal Filter-Based Detection of Microcalcifications. IEEE transactions on biomedical engineering. Vol. 48, pp.1272-1280, (2001).

[20] A. Papadopoulos, D.I. Fotiadis, A. Likas. Characterization of clustered microcalcifications in digitized mammograms using neural networks and support vector machines. Artificial Intelligence in Medicine, Vol. 4, pp.203-220, (2004).

[21] J. Jiang, B. Yao, A.M. Wason. A genetic algorithm design for microcalcification detection and classification in digital mammograms. Computerized Medical Imaging and Graphics, Vol. 31, pp. 4961, (2007).

[22] Helen Seymour, Rosalind Given-Wilson, Louise Wilkinson, Julie Cooke. Resolving Breast Microcalcifications. RG Vol. 20, pp. 307-308, (2000).

[23] Ayman A. AbuBaker, Rami.S.Qahwaji, Stan Ipson, Mammogram Image Segmentation Using Statistical and Morphological based Techniques. 8th Informatics workshop, University of Bradford, (2007).

[24] Guillaume Kom, Alain Tiedeu, Martin Kom. Automated detection of masses in mammograms by local adaptive Thresholding. Computers in Biology and Medicine Vol. 37. pp. 37 - 48. (2007).

[25] M.A. Wirth, D. Nikitenko. Quality evaluation of fuzzy contrast enhancement algorithms. Proc. Annual Meeting of the North American Fuzzy Information Processing Society, (NAFIPS 2005), (2005).

[26] Ayman AbuBaker. Automatic Detection of Breast Cancer Microcalcifications in Digitized X-ray Mammograms. Ph.D., thesis, School of Informatics, University of Bradford-UK. (2008).

[27] Michael Wirth, Matteo Fraschini, and Jennifer Lyon. Contrast enhancement of micro-calcifications in mammograms using morphological enhancement and non-flat structuring elements. Proc. 17th IEEE Symposium on Computer-Based Medical Systems, (CBMS’04), (2004).

[28] Cristian Munteanu and Agostinho Rosa. Gray-Scale Image Enhancement as an Automatic Process Driven by Evolution. IEEE Trans. Systems, man, and cybernetics-part B: Cybernetics, vol. 34, pp. 430-440, (2004).

[29] K. Fukanga, Introduction to Statistical Pattern Recognition. San Diego, CA: Academic, (1990). 
The International Journal of Multimedia \& Its Applications (IJMA) Vol.4, No.5, October 2012

[30]Ayman AbuBaker. Microcalcification Enhancement In Digitized Mammogram Images Using Fuzzy Logic Approach. Ubiqutious computing and communication Journal (UBICC), 7(2), pp: 1255- 1261, (2012).

\section{Author:}

Dr. Ayman A. Abubaker: Asst. Prof. at Electrical and Computer Engineering Department, Applied Science Private University, Amman-Jordan. He got his $\mathrm{PhD}$ in Electronic Imaging and Media Communications (EIMC) from University of Bradford-UK. His main interests are image processing, machine learning, and intelligent mobile robots.

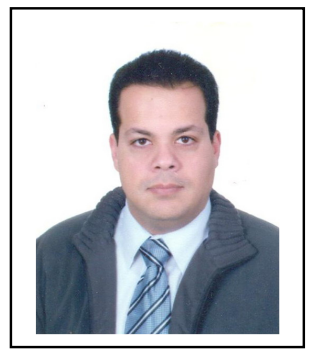

